\title{
The effects of association strength and cross-modal correspondence on the development of multimodal stimuli
}

\author{
Zara P. Y. Chan • Benjamin J. Dyson
}

Published online: 13 November 2014

(C) The Psychonomic Society, Inc. 2014

\begin{abstract}
In addition to temporal and spatial contributions, multimodal binding is also influenced by association strength and the congruency between stimulus elements. A paradigm was established in which an audio-visual stimulus consisting of four attributes (two visual, two auditory) was presented, followed by questions regarding the specific nature of two of those attributes. We wanted to know how association strength and congruency would modulate the basic effect that responding to same-modality information (two visual or two auditory) would be easier than retrieving different-modality information (one visual and one auditory). In Experiment 1, association strengths were compared across three conditions: baseline, intramodal (100\% association within modalities, thereby benefiting same-modality retrieval), and intermodal (100\% association between modalities, thereby benefiting different-modality retrieval). Association strength was shown to damage responses to same-modality information during intermodal conditions. In Experiment 2, association strength was manipulated identically, but was combined with crossmodally corresponding stimuli (further benefiting differentmodality retrieval). The locus of the effect was again on responses to same-modality information, damaging responding during intermodal conditions but helping responding during intramodal conditions. The potential contributions of association strength and cross-modal congruency in promoting learning between vision and audition are discussed in relation to a potential default within-modality binding mechanism.
\end{abstract}

Keywords Multisensory processing $\cdot$ Statistical inference

Z. P. Y. Chan · B. J. Dyson $(\bowtie)$

Department of Psychology, Ryerson University, 350 Victoria Street,

Toronto, Ontario M5B 2K3, Canada

e-mail: ben.dyson@psych.ryerson.ca
Statistical learning refers to the successful registration of associative probability in the environment. It is a process that occurs often without awareness (Kim, Seitz, Feenstra, \& Shams, 2009) and plays a central role in language acquisition, arguably one of the most important instances of human learning. As such, statistical learning has been extensively studied in infants and adults, using both visual and auditory domains (e.g., Fiser \& Aslin, 2002; Saffran, Aslin, \& Newport, 1996). Most recently, statistical learning has been extended to audiovisual cases (e.g., Conway \& Christiansen, 2006; Mitchel \& Weiss, 2011; Robinson \& Sloutsky, 2007; van den Bos, Christiansen, \& Misyak, 2012; Walk \& Conway, 2011), with particular emphasis on the differential constraints associated with learning associations within and between modalities. For example, Conway and Christiansen pointed out that the learning of multiple grammars can benefit from distinct stimulus delivery: learning two grammars within the same dimension (e.g., visual shape) is considerably worse than learning of two grammars either separated across two different attributes within the same modality (e.g., shape and color in the visual domain) or separated across two different attributes of different modalities (e.g., shape in the visual domain and pitch in the auditory domain). Furthermore, the provision of correlated information in a secondary modality might help associative learning in a primary modality (Kawahara, 2007; Mitchel \& Weiss, 2011; Robinson \& Sloutsky, 2007; van den Bos et al., 2012), even when these correlations are arbitrary, such as the relationships between an auditory syllable and visual color (Glicksohn \& Cohen, 2013). In these instances, the use of multiple modalities may serve as a way to make individual grammars more distinctive. Yet, despite these potential benefits, other researchers have observed disadvantages associated with multimodal statistical learning, such as the failure to detect grammar violations, defined by the sequential presentation of triplets divided across multiple modalities (e.g., visual-auditory-visual), relative to triplets defined by a single 
modality (e.g., visual-visual-visual). As Walk and Conway stated: "learning statistical associations between two elements may be more difficult when it takes place across two modalities compared to when it occurs within the same modality." Similar costs in transitioning between stimuli from different modalities have also previously been reported by Spence, Nicholls, and Driver (2001), who showed cross-modal costs (see their "modality-shifting effect") when participants made location discriminations within a block of trials in which targets from one modality were presented more frequently than those from other modalities (e.g., audition $75 \%$, vision $12.5 \%$, and tactile $12.5 \%$ ).

The failure of statistical learning across audition and vision in the case of Walk and Conway (2011) may be attributed to certain paradigmatic choices, such as presenting unimodal stimuli sequentially rather than presenting multimodal stimuli concurrently. Given that many environmental encounters are in fact multimodal (Mitchel \& Weiss, 2011), the use of unimodal stimuli is not as ecologically valid, and it has been shown that multimodal processing is facilitated through temporal (and spatial) coincidence (i.e., concurrent associations rather than sequential associations; Calvert, Spence, \& Stein, 2004; Glicksohn \& Cohen, 2013; Seitz, Kim, van Wassenhove, \& Shams, 2007). To address these concerns, a paradigm used to test object-based learning in both visual (Duncan, 1984) and auditory (Dyson \& Ishfaq, 2008) domains was applied to a unique audio-visual case. To use an auditory example from Dyson and Ishfaq to introduce the paradigm used in the present study, participants were simultaneously presented with two distinct auditory objects: a tone with defined pitch and frequency modulation (FM), and a noise with defined bandpass and amplitude modulation. At each trial, participants were played the composite sound and required to identify two of the four features on the basis of two prompts. The first prompt was presented after stimulus presentation and provided participants with one of the four dimensions and the two options associated with it (e.g., "LOW[pitch]HIGH"). After responding, participants were presented with a second prompt of the same format but related to a different dimension. On half of the trials, the second prompt interrogated the same object by presenting the only other dimension belonging to it. For example, in sameobject trials, if the first prompt interrogated pitch, then the object in question was the tone, and the only other dimension associated with it was FM (e.g., "NO[warble]YES"). On the other half of the trials, the second prompt interrogated the other object by presenting one of the two dimensions belonging to it. For example, in different-object trials, if the first prompt interrogated pitch (belonging to the tone object), then the other object in question was the noise, and the responding could be based on the nature of the bandpass or amplitude modulation. Importantly, both within- and between-object trials involved task switching, and since all trials were randomized, the critical distinction between same- and different-object trials was only apparent during second-prompt responding. The authors hypothesized that if auditory memory for features also incorporated the object from which the feature came (see Duncan, 1984), then performance on the second response should be better for within-object trials than for between-object trials, and this was found to be the case.

Thinking about the shared object benefit led to the interpolation that similar benefits should be observed when assessing multiple pieces of information linked by a shared modality. This maneuver was inspired by previous work (e.g., Benjamins, van der Smagt, \& Verstraten, 2008) that had suggested that some of the constraints associated with processing multiple dimensions and objects are similar to the constraints associated with processing multiple modalities. For example, switching costs between modalities are incurred (e.g., Spence et al., 2001) in the same way that switching costs between dimensions within the same modality are incurred (e.g., Dyson \& Quinlan, 2002), and the joint effect of these costs is subadditive, suggesting some form of interaction (e.g., Sandhu \& Dyson, 2013). Therefore, in the same way that assessing two pieces of information derived from the same object (relative to a different object within the same modality) may be beneficial for processing (Duncan, 1984; Dyson \& Ishfaq, 2008; Hecht, Abbs, \& Vecera, 2008), it follows that assessing two pieces of information derived from the same modality (relative to a different object within a different modality) may also be beneficial for processing. Experiment 1 then extended the assumption of a within-object preference to a within-modality preference (Walk \& Conway, 2011).

Against the backdrop of a within-modality preference, we were interested to see whether this preference might be modulated by associative strength. First, a baseline condition was established in order to assess performance in retrieving two pieces of information from the same modality relative to retrieving two pieces of information from different modalities, with the expectation that performance should be better in the former condition. The likelihood of a feature being associated with any other feature in the baseline condition was equiprobable (probabilistic; $p=.5$ ). Two additional conditions were developed in which the association strength was either deterministic $(p=1)$ or probabilistic $(p=.5)$ with respect to predicting features across the different dimensions. The assumption here was that statistical learning should be facilitated under deterministic conditions (van den Bos et al., 2012). Deterministic associations involved a $100 \%$ match between features from two dimensions: The presentation of Feature A from Dimension 1 was paired with the presentation of Feature $\mathrm{C}$ from Dimension 2 on all trials, and the presentation of Feature B from Dimension 1 was paired with the presentation of Feature D from Dimension 2 on all trials. Probabilistic associations involved only a $50 \%$ match between features from two dimensions: The presentation of Feature A from Dimension 1 was paired with Feature $\mathrm{C}$ from Dimension 2 for 
half of the trials and with Feature D from Dimension 2 for half of the trials. Therefore, depending on whether deterministic associations existed either between or within modalities, this would help or hinder the binding of information across vision and audition. In the intramodal conditions, association strength was deterministic during same-modality trials, but probabilistic during different-modality trials. In this respect, association strength was consistent with the expected within-modality preference. In the intermodal conditions, association strength was deterministic during different-modality trials, but probabilistic during same-modality trials. In this respect, association strength was inconsistent with the expected within-modality preference. As a result, we anticipated that the intramodal condition would further promote same-modality responding, whereas the intermodal condition would lead to the promotion of differentmodality responding, potentially overcoming some of the potential constraints associated with multimodal statistical learning (Walk \& Conway, 2011), and perhaps even reversing the same-modality preference.

\section{Experiment 1}

\section{Method}

Participants Twenty-four undergraduate students (19 female, five male) participated in the study; their mean age was
20.9 years $(S D=5.3)$, and 20 were right-handed. Two additional participants (not included in the final sample) were excluded for error rates exceeding $30 \%$ in any condition. The study was approved for testing by the Research Ethics Board of Ryerson University, and participants received course credit for participation.

Stimuli and apparatus Using SoundEdit 16 (MacroMedia), auditory stimuli of 500-ms high $(523 \mathrm{~Hz})$ and low $(294 \mathrm{~Hz})$ tones were generated with and without FM (modulation frequency $20 \mathrm{~Hz}$, deviation frequency $20 \mathrm{~Hz}$ ) to create the presence or absence of a warble. Then, 5-ms linear onset and offset ramps were also applied in order to avoid click. Monaural sounds were presented from free-field speakers (Harman/ Kardon) positioned on either side of a computer monitor as viewed approximately $57 \mathrm{~cm}$ away, to best simulate spatial coincidence between the auditory and visual stimuli (Calvert et al., 2004). Presenting monaural sounds simultaneously to both left and right speakers allowed for the sound to be heard in an aggregated center space (i.e., the computer monitor; see Kubovy \& Van Valkenburg, 2001, Fig. 11b). Hence, both visual and auditory presentation positions were roughly equated in depth, elevation, and azimuth. Using PowerPoint (Microsoft), we created visual stimuli consisting of squares and diamonds presented in either 2-D or 3-D form. The squares were $3.3 \mathrm{~cm}^{2}$, and the diamonds were created by rotating the squares $45^{\circ}$ counterclockwise (see Fig. 1). Presentation of the
Fig. 1 In Experiment 1, visual stimulus variation consisted of shape (V1) and depth (V2), and auditory stimulus variation consisted of pitch (A1) and warble (A2). In Experiment 2, the visual stimuli varied in shape (V1) and color (V2), and the auditory stimuli again varied in pitch (A1) and warble (A2)

\section{EXPERIMENT 1}
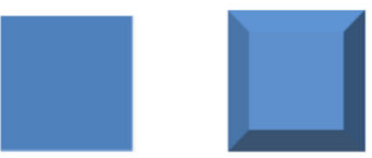

VISUAL

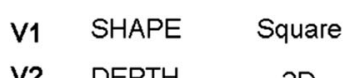

$2 \mathrm{D}$

Square

$3 \mathrm{D}$

Low

A1 PITCH

A2 WARBLE

No

Low

Yes
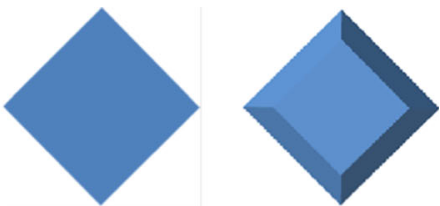

Diamond

$2 \mathrm{D}$

Diamond

3D

High

No

High

Yes
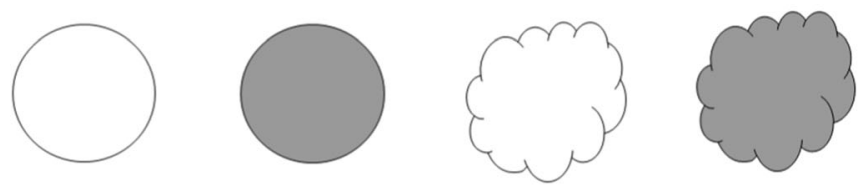

VISUAL

$\begin{array}{ll}\text { v1 } & \text { SHAPE } \\ \text { v2 } & \text { COLOR }\end{array}$

Circle

Circle

Grey

Cloud

Cloud

White

White

Grey

AUDITORY

$\begin{array}{llc}\text { A1 } & \text { PITCH } & \text { Low } \\ \text { A2 } & \text { WARBLE } & \text { No }\end{array}$

Low
Yes

High

No
High

Yes 
stimuli was controlled by PsyScope (Cohen, MacWhinney, Flatt, \& Provost, 1993), and responses were recorded using a PsyScope Button Box. Our confidence that participants could associate audition with vision was increased by the demonstration of successful audio-visual interactions using this setup in previous studies (e.g., Sandhu \& Dyson, 2012; Wilbiks \& Dyson, 2013).

Design Experimental blocks of 128 trials each were developed using combinations of the visual dimensions of shape (V1; square, diamond) and depth (V2; 2-D, 3-D), and the auditory dimensions of pitch (A1; low, high) and warble (A2; no, yes). At each trial, a stimulus consisting of four attributes was presented, and participants were required to respond to the specific nature of only two of the attributes following stimulus presentation and prompting (after Dyson \& Ishfaq, 2008). The response prompts were "SQUARE[shape]DIAMOND" and "2D[depth]3D" for visual stimuli, and "LOW[pitch]HIGH" and "NO[warble]YES" for auditory stimuli. Responses were made by pressing a button (left or right) that corresponded to the feature that participants believed they had previously seen or heard. Responses for the first prompt constituted each feature (e.g., square, diamond) of each dimension (e.g., V1: shape) presented for 16 trials. For same-modality trials, responses for the second prompt were based on the only other dimension belonging to the modality interrogated at the first prompt (e.g., shape followed by depth [or vice versa], in the case of visual responding, and warble followed by pitch [or vice versa], in the case of auditory responding). For different-modality trials, responses to the second prompt were based on only one of the dimensions from the alternative modality (e.g., shape followed by pitch [or vice versa] and warble followed by depth [and vice versa]). The assignments of the associations between modalities were counterbalanced across participants (e.g., V1 paired with $\mathrm{A} 1$ and $\mathrm{V} 2$ paired with $\mathrm{A} 2 \mathrm{vs.} \mathrm{V1} \mathrm{paired} \mathrm{with}$ $\mathrm{A} 2$ and V2 paired with A1).

On the basis of this initial design, three conditions were developed and completed in a counterbalanced order: baseline, intramodal, and intermodal. All of the conditions differed in terms of strengthening or weakening of the associations between stimulus dimensions (see Fig. 2). In the baseline condition, all possible combinations of the stimuli were orthogonal, meaning that neither same- nor different-modality trials had predictive value. In the intramodal condition, there was predictive (deterministic) value for within-modality associations, but no predictive value (probabilistic) for betweenmodality associations. For example, the feature of A1 (e.g., low pitch) would predict the feature of A2 (e.g., no warble) for same-modality trials, but not the feature of V1 (e.g., the shape could be either square or diamond) for different-modality trials. For the intermodal condition, there was predictive (deterministic) value for between-modality associations but no predictive value (probabilistic) for within-modality associations. For example, the feature of A1 (e.g., low pitch) would predict the feature of V1 (e.g., square shape) for differentmodality trials, but not the feature of A2 (e.g., warble could be either present or absence) for same-modality trials. To avoid the additional contribution of response repetition to cases in which the dimensions were associated, half of the participants completed a version of the task in which the associations predicted the opposite responses (e.g., square [a left-button response] would predict either 3-D or high pitch [both rightbutton responses]).

Procedure Participants were given a tutorial on the different stimuli to ensure adequate discrimination before they completed a practice block consisting of 12 trials taken at random from the baseline condition. Participants then completed one block of 128 trials for each of the three counterbalanced conditions (baseline, intramodal, and intermodal). Each trial (see Fig. 3) began with a fixation cross for $500 \mathrm{~ms}$ before an audio-visual stimulus was presented for $500 \mathrm{~ms}$. Following the presentation of a blank screen for $500 \mathrm{~ms}$, the first response prompt appeared and remained onscreen until the participant made a response. After each response, there was a checking period for $50 \mathrm{~ms}$ before participants were given feedback for $450 \mathrm{~ms}$, in the form of a green cross for a correct response or a red cross for an incorrect response. The second response prompt then appeared until response, and similar feedback was provided before the start of the next trial. Following the procedures of previous studies (Dyson \& Ishfaq, 2008), all trials were randomized, so the distinction between same- and different-modality trials was only apparent during the second response prompt.

\section{Results}

The median reaction time (RT) data and mean error rate data were submitted to separate three-way repeated measures analyses of variance (ANOVAs) consisting of the factors Condition (baseline, intramodal, intermodal), Modality (same, different), and Response (first, second) (see Table 1 and Fig. 4). ${ }^{1}$ Greenhouse-Geisser corrections were used to adjust degrees of freedom $(d f \mathrm{~s})$ when the assumption of sphericity had been violated, and Tukey's HSD $(p<.05)$ was used to investigate post-hoc effects. In addition to main effects of modality $(p<.001)$ and response $(p<.001)$, the RT data revealed a significant Modality $\times$ Response interaction $(p<.001)$,

\footnotetext{
${ }^{1}$ Concerns regarding the violation of normality in the data set were allayed by carrying out square-root transformations on both the RT and error rate data (including the addition of a +1 constant to observations for which the error rate was 0 ). The significances of the main effects and interactions generated by this revised ANOVA across both experiments and measures were equivalent to those from our original analysis.
} 
BASELINE

\begin{tabular}{|c|c|c|c|c|}
\hline & V1 & V2 & A1 & A2 \\
\hline V1 & - & .5 & .5 & - \\
\hline V2 & .5 & - & - & .5 \\
\hline A1 & .5 & - & - & .5 \\
\hline A2 & - & .5 & .5 & - \\
\hline
\end{tabular}

INTRA-MODAL

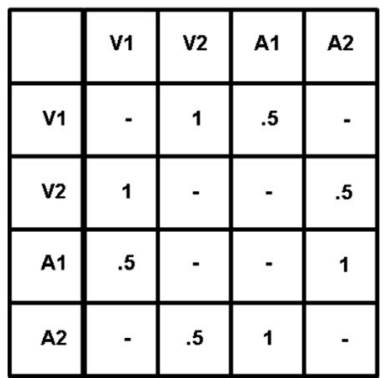

INTER-MODAL

\begin{tabular}{|c|c|c|c|c|}
\hline & V1 & V2 & A1 & A2 \\
\hline V1 & - & .5 & 1 & - \\
\hline V2 & .5 & - & - & 1 \\
\hline A1 & 1 & - & - & .5 \\
\hline A2 & - & .1 & .5 & - \\
\hline
\end{tabular}

Fig. 2 Association mappings in Experiments 1 and 2. In the baseline condition, there was no predictive value for any attribute pairing. In the intramodal condition, attributes had predictive value within the same modality, and in the intermodal condition, attributes had predictive value between modalities. Note that the association between modalities was counterbalanced across participants, such that half of the participants had V1 paired with A1 and V2 paired with A2 (shown here), whereas the other half of the participants had V1 paired with $\mathrm{A} 2$ and V2 paired with A1

again similar to the RT data, different-modality trials were significantly more errorful than were same-modality trials during the baseline ( $12.40 \%$ and $9.53 \%$, respectively) and intramodal ( $11.64 \%$ and $7.61 \%$, respectively) conditions, but not during the intermodal condition $(11.21 \%$ and $11.16 \%$, respectively), where associations were stronger between modalities. Again, the equivalence of performance between same-modality and different-modality trials during intermodal responding suggests that the within-modality preference can be attenuated when associations are deterministic between modalities but probabilistic within modalities. In addition, the error rates for intramodal same-modality trials were significantly smaller than those in all other conditions, apart from baseline same-modality trials.

Most critically, a significant three-way Condition $\times$ Modality $\times$ Response interaction $(p<.001)$ was also shown. As is shown in the bottom half of Fig. 4 , the Modality $\times$ Response interaction is consistent across the baseline and intramodal conditions (in which error rates are largest for second different-modality trials relative to all other cells within that condition), but different in the intermodal condition (in which error rates are larger for both second different-modality and second same-modality trials relative to first responding). In addition to the other noncritical comparisons, we found that second different-modality error rates did not differ significantly across conditions $(15.82 \%$ for baseline, $15.26 \%$ for intramodal, and $13.24 \%$ for intermodal), but second samemodality error rates did differ across conditions $(10.01 \%$ for ence. Regarding the Condition $\times$ Modality interaction, and

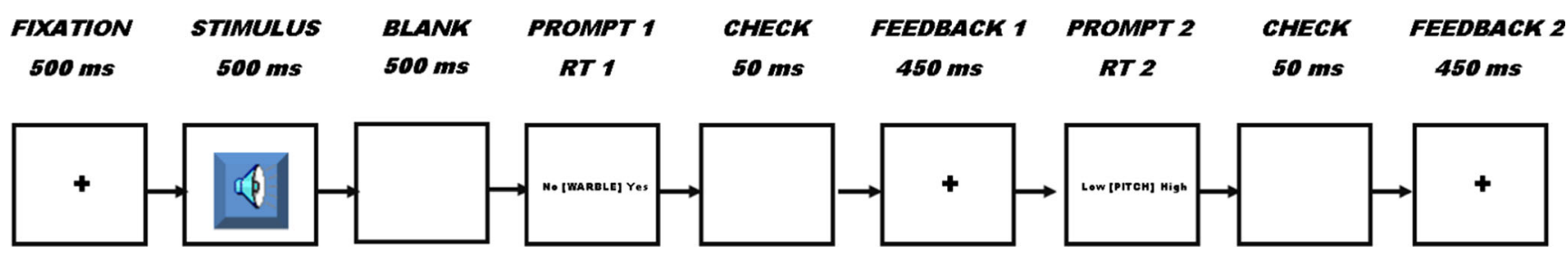

Fig. 3 Schematic of the procedure for Experiments 1 and 2 
Table 1 Summary of the three-way ANOVA for median reaction time and mean error rate data in Experiment 1

\begin{tabular}{|c|c|c|c|c|c|c|c|c|c|}
\hline \multirow[t]{2}{*}{ Metric } & \multirow[t]{2}{*}{$d f$} & \multicolumn{4}{|c|}{ Reaction Time } & \multicolumn{4}{|c|}{ Error Rate } \\
\hline & & $F$ & $M S E$ & $p$ & $\eta_{\mathrm{p}}^{2}$ & $F$ & $M S E$ & $p$ & $\eta_{\mathrm{p}}^{2}$ \\
\hline Condition $(\mathrm{C})$ & 2,46 & 0.51 & 19,336 & .605 & .022 & 1.89 & 36 & .163 & .076 \\
\hline Modality (M) & 1,23 & 17.07 & 11,199 & $<.001$ & .426 & 22.57 & 17 & $<.001$ & .495 \\
\hline Response (R) & 1,23 & 38.34 & 18,818 & $<.001$ & .625 & 45.07 & 25 & $<.001$ & .662 \\
\hline $\mathrm{C} \times \mathrm{M}$ & 2,46 & 8.83 & 3,424 & $<.001$ & .277 & 5.83 & 17 & .006 & .202 \\
\hline $\mathrm{C} \times \mathrm{R}$ & 2,46 & 0.20 & 5,040 & .816 & .009 & 0.17 & 34 & .844 & .007 \\
\hline $\mathrm{M} \times \mathrm{R}$ & 1,23 & 28.73 & 12,955 & $<.001$ & .555 & 17.61 & 18 & $<.001$ & .434 \\
\hline $\mathrm{C} \times \mathrm{M} \times \mathrm{R}$ & 2,46 & 0.10 & 3,798 & .903 & .004 & 6.61 & 18 & .003 & .223 \\
\hline
\end{tabular}

Significant ANOVA terms are in bold. The $d f \mathrm{~s}$ for the error rate $\mathrm{C} \times \mathrm{R}$ interaction were Greenhouse-Geisser corrected to $1.598,36.750$

baseline, $7.47 \%$ for intramodal, and $13.59 \%$ for intermodal), with the difference between the intramodal and intermodal conditions being significant. Therefore, the three-way interaction was largely carried by variation in same-modality trials (white bars in Fig. 4) and an increase in error rates for second responding during the intermodal condition $(13.59 \%)$, where associations were stronger across rather than within modalities, relative to the intramodal condition $(7.47 \%)$, where associations were stronger within rather than across modalities.
Discussion

Experiment 1 established a number of key findings. First, the baseline condition confirmed that difficulties associated with retrieving multiple pieces of information from different objects relative to the same object (see Duncan, 1984; Dyson \& Ishfaq, 2008; Hecht et al., 2008) extend to the retrieval of multiple pieces of information from different modalities, relative to the same modality (Walk \& Conway, 2011). Second, the data show that participants were sensitive to manipulations

\section{BASELINE}

\section{A)}

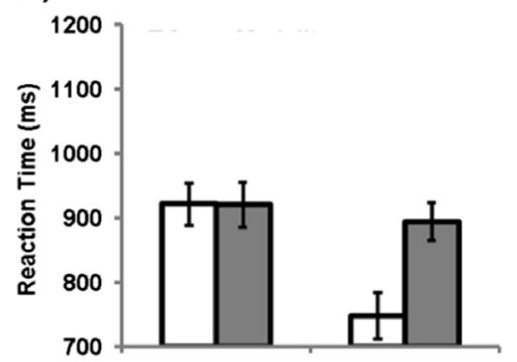

B)

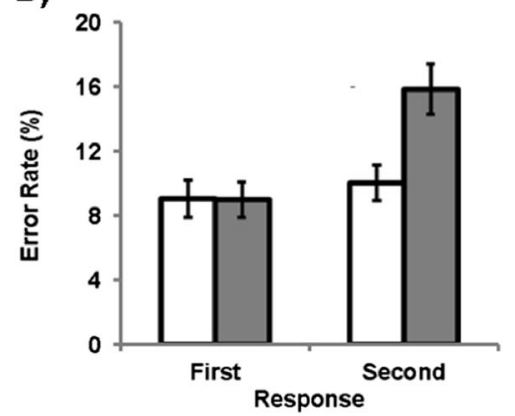

INTRA-MODAL
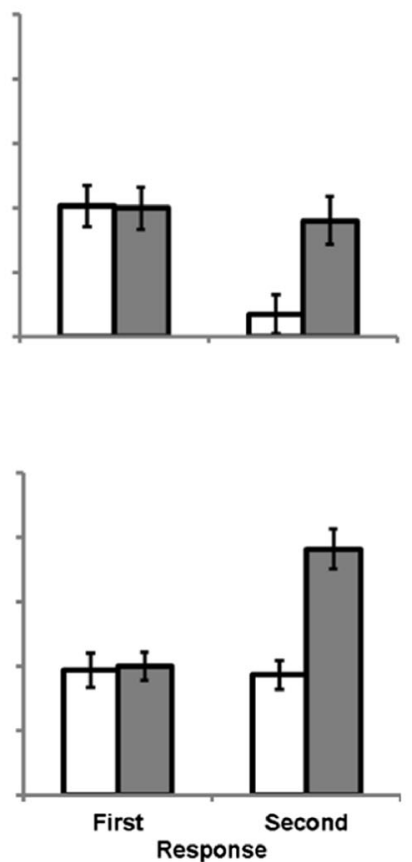

INTER-MODAL

口Same Modality

口Different Modality

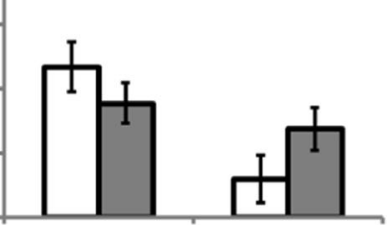

Fig. 4 Graph to show (a) median reaction time and (b) mean error rate data effects in Experiment 1. Error bars represent standard errors. In the case of the reaction time data, a difference between same-modality and different-modality trials is revealed for second responding, extending the within-object effect found in Dyson and Ishfaq (2008) to a within- modality case. The error rate data also reflect this effect, in addition to the observation that strengthening the associations between audition and vision (intermodal condition) served to damage performance in the samemodality trials, relative to the baseline and intramodal conditions 
of association strength, in that error rates were different for second responding between intramodal and intermodal conditions. Third, this effect was driven by same-modality rather than different-modality retrieval, revealing that whereas the strengthening of associations within modalities (intramodal condition) benefited same-modality responding, the strengthening of associations between modalities (intermodal condition) did not benefit different-modality responding, but rather disrupted same-modality responding. Thus, Experiment 1 suggested that the mechanism by which statistical learning occurs cross-modally seems to be via the modulation of the degree of within-modality preference. In Experiment 2, we sought to establish whether the use of cross-modal correspondence would work in a similar way to the use of deterministic association strength in intermodal conditions.

\section{Experiment 2}

In addition to the promotion of multimodal processing via deterministic association strength, the correspondence between auditory and visual values may also play a role (see Melara \& Marks, 1990, Fig. 1; Spence, 2011; Walker \& Walker, 2012, for overviews). For example, structural correspondences such as the brightness of visual stimuli and the loudness of auditory stimuli represent an early form of modality cross-talk, wherein both dimensions are represented by a similar mechanism (such as an increase in neural firing rates) and are thought to require little or no training (Lewkowicz \& Turkewitz, 1980). Later forms of modality cross-talk involve structural and/or semantic components. For example, a picture of a baby crying and a sound of the cries provide semantic congruency, in that both represent the concept of cry, as well as statistical correspondence based on the frequencies of cooccurrence in the environment (Spence, 2011).

In our previous experiment, the visual stimuli consisted of square and diamond shapes in 2-D and 3-D depths, and the auditory stimuli consisted of low and high pitches with or without the presence of a warble. To our knowledge, no preexisting associations existed between these attributes, either intra- or intermodally. To achieve cross-modal correspondence in Experiment 2, the auditory stimuli were as per Experiment 1, but the visual stimuli now varied according to color and shape (see Fig. 1). On the basis of previous work, pitch and lightness were expected to correspond (i.e., higher pitch and lighter color, lower pitch and darker color; Mondloch \& Maurer, 2004), as were warble and shape (i.e., purer tones and rounder shapes, vibrato [warble] tones and more angular shapes; Hossain, 2011). Importantly, we were not aware of any evidence that the pairing of pitch and FM in audition, or the pairing of shape and color in vision, would strengthen within-modality associations. We anticipated that cross-modal correspondences would interact with associative strength in the following ways: In the intramodal condition, association strength and stimulus correspondence would essentially work in opposite directions, since association strength would promote same-modality processing, whereas correspondence would promote different-modality processing. In the intermodal condition, association strength and stimulus correspondence would work together in promoting different-modality retrieval. In the latter condition, we thought it possible that participants might again show a preference for different-modality as opposed to same-modality responding, due to the combination of association strength and stimulus correspondence favoring different-modality retrieval, thereby eliminating the same-modality preference (Walk \& Conway, 2011). Seeking to replicate the findings of Experiment 1, we were once again interested in whether these effects would manifest themselves in the modulation of same-modality or different-modality trials.

\section{Method}

Twenty-four undergraduate students (18 female, six male) participated in the study; their mean age was 21.9 years $(S D=7.3)$, and 22 were right-handed. Two additional participants (not included in the final sample) were excluded for error rates exceeding $30 \%$ in any condition. Experiment 2 was identical to Experiment 1 (see Figs. 1, 2, and 3), apart from the substitution of square and diamond shapes with circle and cloud shapes (V1; circle, cloud), and 2-D and 3-D shapes with white and gray shapes (V2; white, gray). The auditory stimulus attributes remained pitch (A1; low, high) and warble (A2; no, yes).

\section{Results}

The data from Experiment 2 were analyzed in an identical manner to those from Experiment 1 (see Table 2 and Fig. 5). The RT data replicated the main effects of modality $(p<.001)$ and response $(p<.001)$ and the Modality $\times$ Response interaction $(p<.001)$ found in Experiment 1. Here, second samemodality trials $(799 \mathrm{~ms}$ ) showed significant speeding relative to second different-modality trials $(965 \mathrm{~ms})$, first samemodality trials $(974 \mathrm{~ms})$, and first different-modality trials (985 ms). This once again provides evidence for a withinmodality preference. No other pairwise comparisons were significant.

The error rate data revealed main effects of modality $(p=.012)$ and response $(p<.001)$, in addition to a Modality $\times$ Response interaction $(p=.029)$. Second different-modality trials $(12.44 \%)$ produced significantly more errors than did first same-modality trials $(8.16 \%)$, first different-modality trials $(8.49 \%)$, and second same-modality trials $(10.02 \%)$. Withinmodality preference was manifest in terms of the lower error 
Table 2 Summary of the three-way ANOVA for median reaction time and mean error rate data in Experiment 2

\begin{tabular}{|c|c|c|c|c|c|c|c|c|c|}
\hline \multirow[t]{2}{*}{ Metric } & \multirow[t]{2}{*}{$d f$} & \multicolumn{4}{|c|}{ Reaction Time } & \multicolumn{4}{|c|}{ Error Rate } \\
\hline & & $F$ & $M S E$ & $p$ & $\eta_{\mathrm{p}}^{2}$ & $F$ & $M S E$ & $p$ & $\eta_{\mathrm{p}}^{2}$ \\
\hline Condition $(\mathrm{C})$ & 2,46 & 0.38 & 28,571 & .683 & .016 & 1.24 & 46 & .300 & .051 \\
\hline Modality (M) & 1,23 & 45.83 & 12,393 & $<.001$ & .666 & 7.35 & 18 & .012 & .242 \\
\hline Response (R) & 1,23 & 20.28 & 33,835 & $<.001$ & .469 & 29.85 & 20 & $<.001$ & .565 \\
\hline $\mathrm{C} \times \mathrm{M}$ & 2,46 & 0.26 & 4,147 & .776 & .011 & 6.34 & 12 & .004 & .216 \\
\hline $\mathrm{C} \times \mathrm{R}$ & 2,46 & 0.28 & 9,823 & .687 & .012 & 0.95 & 14 & .394 & .040 \\
\hline $\mathrm{M} \times \mathrm{R}$ & 1,23 & 32.58 & 13,124 & $<.001$ & .586 & 5.39 & 15 & .029 & .190 \\
\hline $\mathrm{C} \times \mathrm{M} \times \mathrm{R}$ & 2,46 & 0.07 & 4,423 & .928 & .003 & 4.30 & 14 & .019 & .157 \\
\hline
\end{tabular}

Significant ANOVA terms are in bold. The $d f \mathrm{~s}$ for the error rate $\mathrm{C} \times \mathrm{R}$ interaction were Greenhouse-Geisser corrected to 1.453, 33.417

rate for second same-modality trials relative to second different-modality trials. Additionally in Experiment 2, second same-modality trials produced more errors than did first same-modality trials. No other pairwise comparisons were significant. A Condition $\times$ Modality interaction $(p=.004)$ showed significantly lower error rates associated with intramodal same-modality trials $(7.44 \%)$ relative to all other comparisons, apart from baseline same-modality trials $(9.40 \%)$.

These two-way interactions were modulated by a Condition $\times$ Modality $\times$ Response interaction $(p=.019)$. As in
Experiment 1, in addition to other noncritical comparisons, we found that second different-modality error rates did not differ significantly across conditions $(13.72 \%$ for baseline, $12.70 \%$ for intramodal, and $10.90 \%$ for intermodal). In contrast, second same-modality error rates did differ across conditions (10.65 \% for baseline, $7.32 \%$ for intramodal, and $12.09 \%$ for intermodal), with the difference between the intramodal and intermodal conditions being significant. The direction of this effect was again consistent with the strength of association within each condition: For the intermodal condition, associations between modalities were strengthened,

\section{BASELINE}

\section{A)}

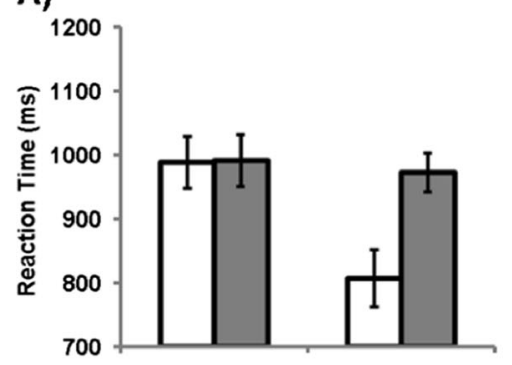

B)

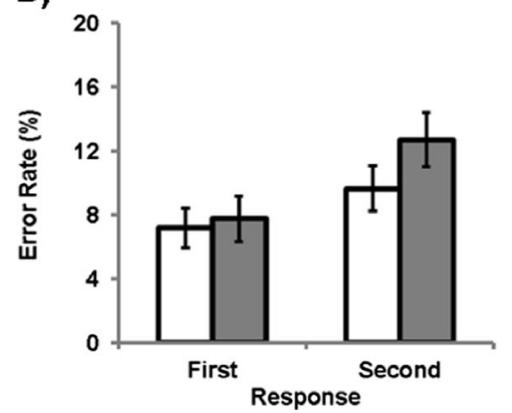

INTRA-MODAL

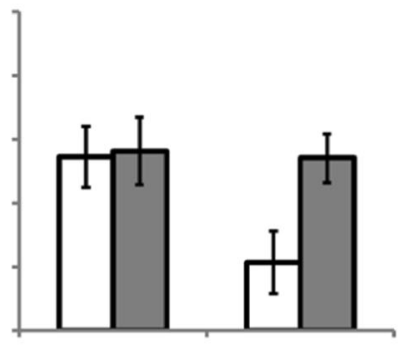

INTER-MODAL

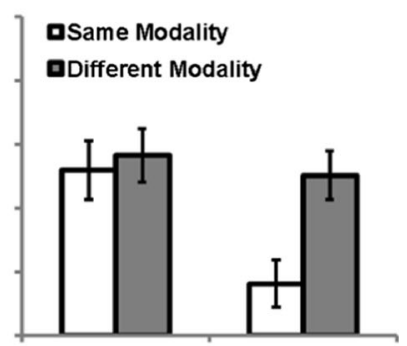

Fig. 5 Graph to show (a) median reaction time and (b) mean error rate data effects in Experiment 2. Error bars represent standard errors. The reaction time data replicate the difference between same-modality and different-modality trials for second responding. The error rate data
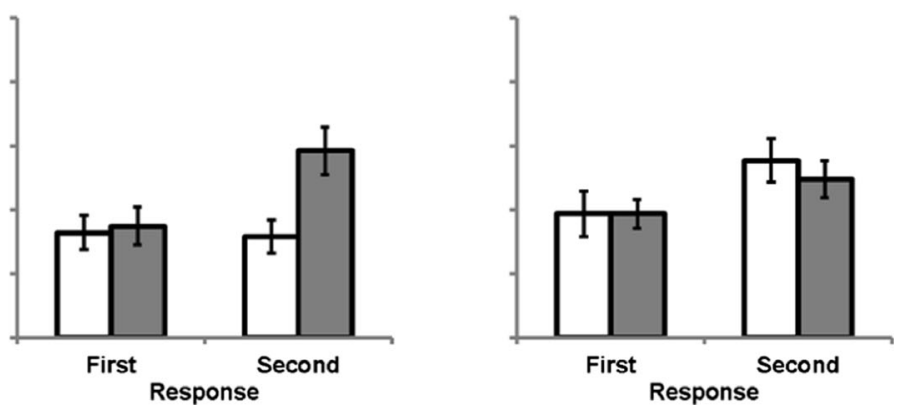

replicate this effect, with the intermodal condition once again being characterized by a deterioration of same-modality performance, as opposed to an improvement in different-modality performance 
relative to the intramodal condition, where associations within modalities were strengthened.

\section{Discussion}

Despite the attempts to introduce the additional factor of cross-modal correspondence to further promote multimodal binding, the data from Experiment 2 replicated the main findings of Experiment 1. Notably, a general preference for the processing of information derived from the same modality as opposed to different modalities was observed in both RTs and error rates, with second responses being both slower and less accurate when retrieval was split across vision and audition, relative to being maintained within a modality. Moreover, participants appeared sensitive to the association strength between stimulus attributes, in that error rates for same-modality responding were reduced when their association strength was deterministic (intramodal condition) rather than probabilistic (intermodal condition). Again, as in Experiment 1 , the effects of association strength were carried by the modulation of same-modality rather than different-modality trials. As a further assessment of the limited contribution of stimulus congruency, cross-experimental analyses confirmed that the error rate data were unchanged by experiments (all interactions with experiment: $p \mathrm{~s}>.124$ ). Although the RT data revealed an interaction between experiment, condition, and modality $(p=.005)$, note that RTs were slower overall in Experiment 2; therefore, this interaction is unlikely to be meaningful. Our failure to find a modulation of audio-visual statistical learning as a function of cross-modal correspondence does not negate previous research that has shown such effects (Glicksohn \& Cohen, 2013), and indeed, design features associated with our particular expressions of crossmodal correspondence might have mitigated the effect (see below).

\section{General discussion}

In the present study, we set out to explore the joint contributions of association strength and cross-modal correspondence on the development of multimodal stimuli. In both Experiments 1 and 2, participants were presented with multidimensional visual and auditory stimuli and were asked to retrieve two pieces of information from either the same modality or different modalities. In accordance with the previous unimodal literature supporting more efficient responding for same- versus different-object performance (e.g., Duncan, 1984; Dyson \& Ishfaq, 2008; Garner, 1976; Hecht et al., 2008), more efficient responding for same-modality versus different-modality performance was established in Experiment 1 (Walk \& Conway, 2011). Importantly, this effect was established in a baseline condition, in which the strength of associations was probabilistic both within and between modalities ( $p s=.5$; van den Bos et al., 2012). Therefore, the data reflect a default type of organization in which information within modalities appears to have preferential links, relative to information between modalities.

With this basic effect established, we additionally sought to investigate whether and how this within-modality preference could be manipulated by associative strength. Using deterministic associations ( $p=1$; van den Bos et al., 2012) for samemodality (intramodal) and different-modality (intermodal) relationships across separate conditions, error rates varied in the expected direction: Performance was more accurate for samemodality trials during an intramodal relative to an intermodal condition. Thus, the ease with which information from the same modality can be categorized is facilitated when the feature of one dimension (e.g., square shape) perfectly predicts the feature of the other dimension (e.g., 2-D depth). However, when the feature of one dimension in one modality (e.g., square shape in vision) perfectly predicts the feature of another dimension in a second modality (e.g., low pitch in audition), the effect is driven not by the facilitation of different-modality responding, but by interference in samemodality responding. The same effects were also observed in Experiment 2, and are important for a number of reasons. First, the data confirm that any inherent preference for learning stimulus relationships within the same modality may be default but not necessarily mandatory (see Yeari \& Goldsmith, 2006, and, Chen \& Cave, 2008, for similar discussions in the context of visual objects). This is evidenced by the equivalence of the error rates for second responding between sameand different-modality trials in intermodal conditions (see Figs. 4 and 5). Second, attempts to facilitate betweenmodality processing were revealed not by improvements in between-modality responding, but by degradation in samemodality responding. This suggests a potentially interesting cognitive constraint, in that the inability to rely on the strength of association within the same modality (as in the intermodal condition) does not result in the compensatory reliance on associations between different modalities. In this respect, processes related to same- and different-modality responding are not necessarily antagonistic, and may in fact be independent from one another. This finding is also notable due to the demonstration of associative (statistical) learning through nontraditional means. First, one might have expected second responses on different-modality trials in the intermodal condition to be improved as a result of statistical optimization and/or cross-modal correspondence, but the effects were manifest by worsening second responses on same-modality trials during intermodal responding. Evidence of statistical learning is usually characterized by improvement in the learned domain rather than by deterioration in the unlearned domain. In this respect, the findings provide evidence for an alternative 
look at manifestations of learning. Second, the paradigm integrated traditionally separated familiarization and test phases (e.g., Turk-Browne, Isola, Scholl, \& Treat, 2008). Usually, participants are exposed to statistical patterns in a primary phase of the study and then tested on the familiarity of both old and new patterns in a second phase of the study. By using a continuous categorization task in the present paradigm, we provided a conservative estimate of learning. This is because at the start of the condition, participants would only be acquiring statistical associations between features, and only at the end of the condition would some of these associations be registered. Therefore, by averaging performance across the entire condition, we probably included trials in which learning had not yet begun. However, a comparison of the first- and second-half performance for error rates in the intermodel condition in Experiment 2 failed to reveal significant effects for condition half (all $p \mathrm{~s}>.130$ ).

A second aim of the experiments was to examine the additional contribution of cross-modal correspondence (e.g., Spence, 2011; Walker \& Walker, 2012) in promoting learning across modalities. To this end, Experiment 2 took advantage of the potential relationships between auditory pitch and visual brightness (Mondloch \& Maurer, 2004) and between auditory warble and visual shape (Hossain, 2011). The absence of any additional contribution from these cross-modal correspondences is curious, given some previous research suggesting that even an arbitrary relationship between vision and audition may be sufficient to bootstrap associations across modalities (van den Bos et al., 2012). However, there are a number of possible reasons for the absence of this effect. First, it appeared that relatively large-scale differences between stimulus attributes (e.g., visual size and auditory pitch) must be in place before relatively small cross-modal correspondence effects can be observed (e.g., $15 \mathrm{~ms}$, in the case of Evans \& Treisman, 2010). This reminds us that establishing cross-modal associations can be rather difficult (Conway \& Christiansen, 2006; Walk \& Conway, 2011) and that the discriminability of the various levels of each stimulus dimension may need to be examined in closer detail. It is possible that even though participants could distinguish between the various levels of the stimulus dimensions used in the present study, the associations were not salient enough to create links between vision and audition. The seminal work of Garner (1976) and the distinction between separable and integral combinations of dimensions will be germane in future work. In particular, using within-modality relations that formally lack interaction (separable) and using between-modality relations that do show evidence of interaction (integral) may go some way toward further promoting the development of multimodal objects (Dyson \& Quinlan, 2010; Grau \& Kemler Nelson, 1988). Second, not all participants were presented with cases of cross-modal correspondence in Experiment 2. As we noted in the design, the between-modality associations were counterbalanced, such that half of the participants experienced pitch-shape and warble-brightness pairs. These are two cross-modal pairings that, to our knowledge, have no known correspondence. Consequently, the effect of congruency in Experiment 2 was weaker than in traditional manipulations of cross-modal correspondence and served as a more conservative measure of cross-modal congruency. Future studies should consider the ways in which cross-modal correspondence can be achieved without response-based accounts (such as when stimulus associations are orthogonal to responding). Despite a broad match on the depth and elevation dimensions, concern could also be raised over a potential discrepancy on the azimuth (visual stimuli presented centrally on a computer screen, auditory stimuli presented peripherally via external speakers). Given the complexity of the visual stimuli required for this study, computer monitor presentation was deemed necessary. Yet although presenting monaural sounds simultaneously to both left and right speakers should result in the sounds being heard in an aggregated center space, precise 3-D spatial congruency has been established in previous studies with simple visual stimuli such as light-emitting diodes built into an array of speakers (see Kristjánsson, Thorvaldsson, \& Kristjánsson, 2014; Spence \& Driver, 1997). Cross-modal correspondence might also be strengthened by improving the spatial correspondence between auditory and visual signals. Nevertheless, the data above show that the promotion of statistical regularities across vision and auditory might not actually facilitate between-modality processing, but might actually damage a default, but not mandatory, preference for the binding of within-modality information.

Author note B.J.D. was supported by an Early Researcher Award granted by the Ontario Ministry of Research and Innovation. The data were presented at the Annual Meeting of the Psychonomic Society, November 14-17, 2014, Toronto, Canada.

\section{References}

Benjamins, J. S., van der Smagt, M. J., \& Verstraten, F. A. (2008). Matching auditory and visual signals: Is sensory modality just another feature? Perception, 37, 848-858.

Calvert, G. A., Spence, C., \& Stein, B. E. (2004). The handbook of multisensory processing. Cambridge: MIT Press.

Chen, Z., \& Cave, K. R. (2008). Object-based attention with endogenous cuing and positional certainty. Perception \& Psychophysics, 70, 1435-1443. doi:10.3758/PP.70.8.1435

Cohen, J., MacWhinney, B., Flatt, M., \& Provost, J. (1993). PsyScope: An interactive graphic system for designing and controlling experiments in the psychology laboratory using Macintosh computers. Behavior Research Methods, Instruments, \& Computers, 25, $257-$ 271. doi:10.3758/BF03204507

Conway, C. M., \& Christiansen, M. H. (2006). Statistical learning within and between modalities: Pitting abstract against stimulus-specific representations. Psychological Science, 17, 905-912. doi:10.1111/j. 1467-9280.2006.01801.x 
Duncan, J. (1984). Selective attention and the organization of visual information. Journal of Experimental Psychology: General, 113, 501-517. doi:10.1037/0096-3445.113.4.501

Dyson, B. J., \& Ishfaq, F. (2008). Auditory memory can be object-based. Psychonomic Bulletin \& Review, 15, 409-412. doi:10.3758/PBR. 15.3.409

Dyson, B. J., \& Quinlan, P. T. (2002). Within- and between-dimensional processing in the auditory modality. Journal of Experimental Psychology: Human Perception and Performance, 28, 1483-1498. doi:10.1037/0096-1523.28.6.1483

Dyson, B. J., \& Quinlan, P. T. (2010). Decomposing the Garner interference paradigm: Evidence for dissociations between macro- and micro-level performance. Attention, Perception, \& Psychophysics, 72, 1676-1691. doi:10.3758/APP.72.6.1676

Evans, K. K., \& Treisman, A. (2010). Natural cross-modal mappings between visual and auditory features. Journal of Vision, 10(1), 6.112. doi: $10.1167 / 10.1 .6$

Fiser, J., \& Aslin, R. N. (2002). Statistical learning of higher-order temporal structure from visual shape sequences. Journal of Experimental Psychology: Learning, Memory, and Cognition, 28, 458-467. doi:10.1037/0278-7393.28.3.458

Garner, W. R. (1976). Interaction of stimulus dimensions in concept and choice processes. Cognitive Psychology, 8, 98-123.

Glicksohn, A., \& Cohen, A. (2013). The role of cross-modal associations in statistical learning. Psychonomic Bulletin \& Review, 20, 1161-1169.

Grau, J. W., \& Kemler Nelson, D. G. (1988). The distinction between integral and separable dimensions: Evidence for the integrality of pitch and loudness. Journal of Experimental Psychology: General, 117, 347-370. doi:10.1037/0096-3445.117.4.347

Hecht, L. N., Abbs, B., \& Vecera, S. P. (2008). Auditory object-based attention. Visual Cognition, 16, 1109-1114.

Hossain, S. (2011). Shapes and sounds: An exploration of audiovisual cross modality. Unpublished manuscript, Dallas, TX: University of Texas.

Kawahara, J.-I. (2007). Auditory-visual contextual cuing effect. Perception \& Psychophysics, 69, 1399-1408. doi:10.3758/ BF03192955

Kim, R., Seitz, A., Feenstra, H., \& Shams, L. (2009). Testing assumptions of statistical learning: Is it long-term and implicit? Neuroscience Letters, 46, 145-149.

Kristjánsson, T., Thorvaldsson, T. P., \& Kristjánsson, Á. (2014). Divided multimodal attention: Sensory trace and context coding strategies in spatially congruent auditory and visual presentation. Multisensory Research, 27, 91-110.

Kubovy, M., \& Van Valkenburg, D. (2001). Auditory and visual objects. Cognition, 80, 97-126.

Lewkowicz, D. J., \& Turkewitz, G. (1980). Cross-modal equivalence in early infancy: Auditory-visual intensity matching. Developmental Psychology, 16, 597-607.

Melara, R. D., \& Marks, L. E. (1990). Interaction among auditory dimensions: Timbre, pitch, and loudness. Perception \& Psychophysics, 48, 169-178.

Mitchel, A. D., \& Weiss, D. J. (2011). Learning across senses: Crossmodal effects in multisensory statistical learning. Journal of
Experimental Psychology: Learning, Memory, and Cognition, 37, 1081-1091. doi:10.1037/a0023700

Mondloch, C. J., \& Maurer, D. (2004). Do small white balls squeak? Pitch-object correspondences in young children. Cognitive, Affective, \& Behavioral Neuroscience, 4, 133-136. doi:10.3758/ CABN.4.2.133

Robinson, C. W., \& Sloutsky, V. M. (2007). Visual statistical learning: Getting some help from the auditory modality. Paper presented at the 29th Meeting of Cognitive Science Society, August 1-4, Nashville, TN

Saffran, J. R., Aslin, R. N., \& Newport, E. L. (1996). Statistical learning by 8-month-old infants. Science, 274, 1926-1928. doi:10.1126/ science.274.5294.1926

Sandhu, R., \& Dyson, B. J. (2012). Re-evaluating visual and auditory dominance through modality switching costs and congruency analyses. Acta Psychologica, 140, 111-118. doi:10.1016/j.actpsy.2012. 04.003

Sandhu, R., \& Dyson, B. J. (2013). Modality and task switching interactions using bi-modal and bivalent stimuli. Brain and Cognition, 82, 90-99.

Seitz, A. R., Kim, R., van Wassenhove, V., \& Shams, L. (2007). Simultaneous and independent acquisition of multisensory and unisensory associations. Perception, 36, 1445-1453.

Spence, C. (2011). Crossmodal correspondences: A tutorial review. Attention, Perception, \& Psychophysics, 73, 971-995. doi:10. 3758/s13414-010-0073-7

Spence, C., \& Driver, J. (1997). Audiovisual links in exogenous covert spatial orienting. Perception \& Psychophysics, 59, 1-22. doi:10. 3758/BF03206843

Spence, C., Nicholls, M. E. R., \& Driver, J. (2001). The cost of expecting events in the wrong sensory modality. Perception \& Psychophysics, 63, 330-336. doi:10.3758/BF03194473

Turk-Browne, N. B., Isola, P. J., Scholl, B. J., \& Treat, T. A. (2008). Multidimensional visual statistical learning. Journal of Experimental Psychology: Learning, Memory, and Cognition, 34, 399-407. doi:10.1037/0278-7393.34.2.399

van den Bos, E., Christiansen, M., \& Misyak, J. B. (2012). Statistical learning of probabilistic nonadjacent dependencies by multiple-cue integration. Journal of Memory and Language, 67, 507-520.

Walk, A. M., \& Conway, C. M. (2011). Multisensory statistical learning: Can associations between perceptual categories be acquired? Boston, MA: Paper session presented at the meeting of Cognitive Science Society.

Walker, P., \& Walker, L. (2012). Size-brightness correspondence: Crosstalk and congruity among dimensions of connotative meaning. Attention, Perception, \& Psychophysics, 74, 1226-1240. doi:10. 3758/s13414-012-0297-9

Wilbiks, J. M. P., \& Dyson, B. J. (2013). Effects of temporal asynchrony and stimulus magnitude on competitive audio-visual binding. Attention, Perception, \& Psychophysics, 75, 1883-1891. doi:10. 3758/s13414-013-0527-9

Yeari, M., \& Goldsmith, M. (2006). Is object-based attention mandatory? Visual Cognition, 15, 119-123. 